and usually very extensive evaluation that occurs in clinical research practice compared with standard medical practice. $\mathrm{He}$ does not consider that these aspects of placebo response might be inherent in the environment of clinical trials, but are not generalisable to day-to-day general or psychiatric practice (although these differences are difficult to assess quantitatively). Other factors of the placebo response in clinical research are potentially amenable to change. These include potentially unhelpful-toresearch, overly encouraging behaviours of those conducting the clinical trial, and false, overly optimistic patient assumptions and expectations about their outcome in the research trial, leading to inaccurate reporting of symptoms and thereby excessive response in those patients randomised to placebo. It is likely that increased efforts in patient and investigator education about how both patients and study site staff can be helpful in forming a productive research alliance and not generate 'wishful thinking' and the overly positive responses that might accompany it, is needed to reduce the costly, wasteful number of failed trials caused by excessive placebo response. In response to this need, I have recently proposed PREECT (Patient and Rater Education about Expectations in Clinical Trials), a two-component approach to the problem (Zimbroff, 2001). Briefly, the first component focuses on ensuring that clinical trial participants understand that they are entering a research alliance - not receiving regular medical care. The second component involves education of study site staff. Both anecdotal feedback and data lend support to the contention that PREECT reduces placebo response rates and, thus, could ultimately reduce the numbers of patients needed in placebo-controlled trials to achieve sufficient power to test the null hypothesis. Further exploration and validation of the PREECT approach would benefit patients and researchers involved in antidepressant trials, and would reduce the likelihood of an excessive placebo response.

Finally, a critique of the scales used as primary efficacy measures needs to be considered. This in itself is a thesis. The very fact that regulatory authorities in the USA are collaborating with researchers on tackling this issue lends support to its importance as a consideration in appraising this topic.

Andrews, G. (200I) Placebo response in depression: bane of research, boon to therapy. British Journal of Psychiatry, 178, 192-194.
Leber, P. D. (1989) Hazards of inference: the active control investigation. Epilepsia, 30 (suppl. I), S57-S63.

(1991) The future of controlled clinical trials. Psychopharmacology Bulletin, 27, 3-8.

World Medical Association Declaration of Helsinki (2000) Ethical Principles for Medical Research Involving Human Subjects. http: / /www.wma. net/e/policy/ 17-c_e.htm

Zimbroff, D. L. (200I) Patient and Rater Education of Expectations in Clinical Trials (PREECT). Journal of Clinical Psychopharmacology, in press.

D. L. Zimbroff Pacific Clinical Research,

San Bernardino, California, USA

\section{Cannabis regimes}

We read with interest MacCoun \& Reuter's (2001) report on evaluating alternative cannabis regimes. In The Netherlands, drug policy is a topic of great interest. A scientific evaluation of policy regimes would be highly appreciated. MacCoun \& Reuter's paper illustrates that this, however, is not an easy job.

As the authors stress, cross-national scientific evaluations are hampered by a lack of comparability due to methodological differences. Nevertheless, many studies summarised in their Table 1 are not methodologically comparable. MacCoun \& Reuter compare the results of Dutch school surveys with those of population surveys in the USA. However, school surveys yield higher prevalence figures of substance use than population surveys do (Gfroerer et al, 1997). Furthermore, the age group "approximately 18 " from the Dutch school survey is compared with the 18-year-old age group in the American national study. In The Netherlands schooling is compulsory until the age of 15-16 years, so 18-year-old high school students cannot be considered as representative of all 18-year-olds in our country. Among high school students aged 12-18 years we saw an increase in cannabis use in 1984-1996, but this had stabilised in 1999. The arguments that the rise may be associated with the coffee shop model and with a phenomenon the authors describe in terms of commercialisation and glamorisation do not quite convince us.

(a) The increase in cannabis prevalence coincides with a supposed increase in the number of coffee shops but this does not prove a causal relationship.

(b) About $80 \%$ of Dutch municipalities have no coffee shops at all (Bieleman $\&$ Goeree, 2000). Less than half of cannabis consumers purchase the drug in a coffee shop - the majority obtains it elsewhere (from a friend, a private house, sale on the street, courier services and take-away services).

(c) The authors do not present clear definitions of the concepts commercialisation and glamorisation. Coffee shops must adhere to the so-called AHOJ-G criteria, which include no advertising. The Public Prosecution Department proclaimed deviation from these criteria a nationwide criminal prosecution policy in 1991.

(d) The increase in cannabis use in the USA seems to have taken place much earlier than in Europe. The authors do not offer a plausible explanation for this trend but indicate the importance of non-policy factors.

(e) Countries with a high prevalence of drug use are more likely to experience a downward trend than countries with low prevalence figures. This is now the case in Europe: an ongoing increase in countries with previously low use levels, and stabilisation or even decline in countries with previous high prevalence figures, both in general population studies and in school surveys, confirm the tendency towards convergence (European Monitoring Centre for Drugs and Drug Addiction, 2000; Hibell et al, 2000). In the UK cannabis use among students was significantly lower in 1999 than in 1995, while in France cannabis prevalence increased steeply, although there are no coffee shops in France. In The Netherlands, among students between 1996 and 1999, not only cannabis use but also use of ecstasy, cocaine, heroin and amphetamines stabilised (de Zwart et al, 2000).

Clearly, trends in drug use are influenced by a complex interplay of factors.

Bieleman, B. \& Goeree, P. (2000) Coffeeshops Geteld, Aantal Verkooppunten van Cannabis in Nederland. Groningen/Rotterdam: Bureau Intraval.

European Monitoring Centre for Drugs and Drug Addiction (2000) Annual Report 2000. Lisbon: EMCDDA

Gfroerer, J., Wright, D. \& Kopstein, A. (1997) Prevalence of youth substance use: the impact of methodological differences between two national surveys. Drug and Alcohol Dependence, 47, 19-30.

Hibell, B., Andersson, B., Ahlström, S., et al (2000) The 1999 ESPAD Report: Alcohol and Other Drug Use Among Students in 30 European Countries. Stockholm: Swedish Council for Information on Alcohol and Other Drugs, and the Council of Europe Pompidou Group. 
MacCoun, R. \& Reuter, P. (200I) Evaluating alternative cannabis regimes. British Journal of Psychiatry, 178, $123-128$

de Zwart, W. M., Monshouwer, K. \& Smit, F. (2000) Cannabis use among Dutch students has stabilised. http: //www.trimbos.nl

W. de Zwart, M. van Laar Trimbos-institute, Netherlands Institute of Mental Health and Addiction, PO 725, 3500 AS Utrecht, The Netherlands

\section{Effects of schizophrenia on patients' relatives}

Tennakoon et al (2000) stated that their study is 'one of the first' to investigate burden among caregivers of people with first-episode psychosis. Their paper was published 13 years after our paper which covered much the same ground (The Scottish Schizophrenia Research Group, 1987) and which they did not mention.

We found, using the General Health Questionnaire (GHQ), that $24(77 \%)$ of 31 main caregivers were categorised as 'psychiatric cases'; this compares with $12 \%$ of caregivers in the Tennakoon et al study, which also used the GHQ. However, the relatives in our study were interviewed during the first week of the patients' first admission to hospital, and before the patients received antipsychotic medication. Tennakoon et al's patients could have been ill for up to 2 years and received up to 12 weeks of antipsychotic drugs - hardly 'first-episode'.

In our 5-year follow-up study (The Scottish Schizophrenia Research Group, 1992), 14 of the 19 relatives who were still living with the patient were reassessed; 6 $(43 \%)$ were still categorised as 'cases', using the GHQ. We concluded that a patient's illness had a considerable and continuing effect on his or her relatives.

The Scottish Schizophrenia Research Group (1987) The Scottish First Episode Schizophrenia Study IV.

Psychiatric and social impact on relatives. British Journal of Psychiatry, 150, 340-344.

- (1992) The Scottish First Episode Schizophrenia Study VIII. Five-year follow-up: clinical and psychosocial findings. British Journal of Psychiatry, 16I, 496-500.

Tennakoon, L., Fannon, D., Doku, V., et al (2000) Experience of caregiving: relatives of people experiencing a first episode of psychosis. British Journal of Psychiatry, 177, 529-533.

R. G. McCreadie Department of Clinical Research, Crichton Royal Hospital, Dumfries DGI 4TG

\section{Over-representation of Black people in secure psychiatric facilities}

I read with great interest Lelliot et al's (2001) survey of patients from an innerLondon health authority in medium secure psychiatric care. In particular, the authors set out to compare Black and White patients and found statistical differences which they dismiss. The scores on the Health of the Nation Outcome Scales and compound variables of clinical behaviour and social function differed between Black and White patients, but Lelliot et al comment that these may not be clinically significant.

If statistically significant findings on scales used in the study need not be explained, then the instruments cannot be considered valid to address the third aim of the study, to compare Black and White patients. Black patients were significantly less morbid on a number of clinical, social and behavioural variables, including affective symptoms, activities of daily living, problems with living skills, relationship problems and other mental or behavioural problems of self-harm and overactive and aggressive behaviour. Why should Black patients with less severe psychopathology or aggressive behaviour continue to find themselves in medium secure units?

There are two possible explanations. One is non-engagement with treatment options in less secure environments. It is known that Black patients are more likely to abscond from in-patient units (Falkowski et al, 1990) and that they are increasingly dissatisfied with each consecutive in-patient admission (Parkman et al, 1997).

An alternative explanation is that Black patients are perceived to be more dangerous despite lower ratings of psychopathology (Cope, 1990; Lewis et al, 1990). Lelliot et al unfortunately dismiss important findings as clinically insignificant. These very findings warrant further research and exploration and such work may well deliver a better understanding of why Black people are over-represented in secure psychiatric facilities.

Cope, R. (1990) Psychiatry, ethnicity \& crime. In Forensic Psychiatry (eds R. Bluglass \& P. Bowden). London: Churchill Livingstone.

Falkowski, J., Watts, V., Falkowski, W., et al (1990) Patients leaving hospital without the knowledge or permission of staff - absconding. British Journal of Psychiatry, 156, 488-490.

Lelliott, P., Audini, B. \& Duffett, R. (200I) Survey of patients from an inner-London health authority in medium secure psychiatric care. British Journal of Psychiatry, 178, 62-66.

Lewis, G., Croft-Jeffreys, C. \& David, A. (1990) Are British psychiatrists racist? British Journal of Psychiatry, I57, 4I0-4I5.

Parkman, S., Davies, S., Leese, M., et al (1997) Ethnic differences in satisfaction with mental health services among representative people with psychosis in South London: PRiSM Study 4. British Journal of Psychiatry, I7I, 260-264.

K. Bhui Department of Psychiatry,

St Bartholomew's and The Royal London School of Medicine and Dentistry, Queen Mary \& Westfield College, University of London, Mile End Road, London El 4NS

\section{Evidence-based psychiatry}

Lawrie et al (2001) have touched upon the core problem of evidence-based psychiatry by raising the most relevant questions. Although the evidence-based medicine movement began in 1992 (Sackett et al, 2000), it was not until the Royal College of Psychiatrists introduced a Critical Review Paper to the MRCPsych Part II examination in 1999 that clinicians suddenly realised the problems of not knowing enough about critical appraisal of scientific papers.

Brown \& Wilkinson (2000) assert, "Psychiatrists should be able to evaluate published literature both in terms of its scientific validity and its clinical relevance". Why - to be able to practise evidence-based psychiatry, or to help trainees pass their exam? In a Scottish survey Lawrie et al (2000) discovered that senior psychiatrists found the time required to search and appraise the literature as the greatest barrier to practising evidence-based psychiatry. Would they be able to practise better if they had enough time, for instance 60 minutes per day? I do not know how to search for the best evidence to answer this question. I have recently read the recommended books (Brown \& Wilkinson, 2000; Sackett et al, 2000), and I have also attended a few evidence-based medicine workshops. When my patients and trainees ask reallife clinical questions, I often get lost. Is this the beginning of my ageing-related cognitive impairment? Or is it just because I am such a busy clinician that I do not have time and need to take evidence-based psychiatry seriously? But how can we help our trainees? What about our own revalidation? We cannot really fudge the issue any more if we want to remain effective trainers. 